Qi-huo Wei

C. Bechinger

D. Rudhardt

P. Leiderer

\section{Structure of two-dimensional colloidal systems under the influence of an external modulated light field}

Qi-huo Wei-Dr, C. Bechinger (メ0)

D. Rudhardt · P. Leiderer

Fakuläl für Physik

Lehrstuhl Prof. Dr. P. Lciderer

University of Konstanz

D-78434 Konstanz

Germany

E-mail: Clemens.Bechinger@uni-

konstanz.de

\author{
Abstract The presence of a mod- \\ ulated laser field can induce \\ crystallization of a colloidal liquid \\ where the particles interact via \\ screened Coulomb repulsion. This \\ phenomenon is called laser-induced \\ freezing (LIF). In this paper, we \\ present experimental results on LIF \\ which were performed under con- \\ trolled particle interaction potentials.
}

This was achieved by defined ion concentration conditions during our experiments. We observed distorted and almost perfect hexagonal siruciures as well as a modulated liquid as a function of the periodicity of the modulated laser field.

Key words Laser-induced freezing and melting colloidal dispersion

\section{Introduction}

Since the pioneering work by Ashkin and co-workers on optical forces acting on small dielectric particles [1], there has been an enormous interest in the field of particle manipulation with light fields. One example are optical tweezers which allow to trap and manipulate single or several particles with one or more intense laser beams. Light forces can also be used to measure interaction forces between colloidal particles and motor molecules [2-5], to probe the elasticity of single polymer like DNA [6], or to investigate properties of membranes $[7,8]$.

It has been also demonstrated, that by creating an extended light intensity pattern, c.g. by interfering two or more laser beams, the structure of many colloidal particles can be manipulated $[9,10]$. For colloids with effectively hard sphere interaction, one can organize the particles to form any structure, even a two-dimensional fivefold sym. metrical and three-dimensional ones, according to the structure of intensity antinodes where the particles are trapped $[9,10]$. For strongly interacting charge-stabilized colloidal particles where the interparticle interaction is a screened Coulomb potential, it has been shown experimentally by Chowdhury et al. [9] that a rwo-dimensional colloidal liquids starts to crystallize when exposed to a periodic light pattern created by two interfering laser beams. When the wave vector of the modulation potential is chosen to coincide the location of the first peak of the structure factor of colloidal liquids (or the periodicity $d=\sqrt{3 a} / 2$, with the mean interparticle separation), a dominantly hexagonal order is observed. This effect is called laser-induced freezing (LIF) $[9,11]$.

Later, density functional theory and Monte-Carlo simulations confirmed the existence of LIF and also predicted that this freezing transition changes from a first order to second order one via a tricritical point. Furthermore, it is expecled that a colloidal crysial can re-melt (LIM) when the external field exceeds some critical value $[12,13]$. This, however, bas not been proven experimentally, yet.

LIF is anyway a result of many-body effects, although one can understand it in the following way, that the external potential induces the alignment of the particles along rows, whereas the interparticle-screened Coulomb repulsion leads to an equal distribution of particles within a single row (see Fig. 2c) and to the registration of particles 
in neighboring rows. As shown by density functional theory [12]. LIF is, in fact, the excitation of the density modulation modes in colloidal liquids with one specific external modulation potential. In this paper we periorm experiments to study the structure when the periodicity (or the wave vector) of the external modulation potential deviates from the above value $(\sqrt{3 a} / 2)$. To control the ion concetration we exployed a continuous deionization technique [14] which allows us to adjust different salt concentrations. In the following we present the results on LIF and the induced structures.

\section{Experimental}

The sample cell is composed of two microscopic cover glasses whose spacing can be adjusted from several $\mathrm{mm}$ to about $20 \mu \mathrm{m}$. After assembling the cell, it was connecled 10 a closed circuit which contained the colloidal suspension. We used charge-stabilized surfactant-free polystyrene sulfate particles from IDC with a diamerer of $3 \mu \mathrm{m}$. The particle concentration was about $1.5 \times 10^{7} / \mathrm{ml}$, but due to sedimentation the actual particle concentration in the cell is assumed to be somewhat higher. The suspension was then pumped througl this circuit which also contained a vessel of ion exchanger and an electrical conductivity probe to control the ionic strength in the suspension. This method allowed us to perform measurements at different ionic strengths [14].

Figure 1 shows schematically the setup used in our experiments. The beam of an argon ion laser (TMoo mode, $\lambda=514 \mathrm{~nm}, I_{\max }=2.6 \mathrm{~W}$ ) is split into two parallel beams of equal intensity by means of two beam splitters (BSI, BS2) and two mirrors (M1, M2). The distances $s$ of the parallel beams can be adjusted by the position of the mirror M2 which is mounted on a motor controlled translation stage. After passing the lens $\mathrm{L}$, the two beams are overlapped inside the sample cell where they produce interference fringes. The spacing of the interference fringes $d$ is controlled by beam spacing $s$ through

$d=\lambda / 2 \sin (\theta / 2)=f / / s$,

where $\theta$ is the angle between the laser beams and $f$ the focus length of the lens $\mathrm{L}$. The sample ceil with the colloidal suspension is illuminated with white lighr (not shown in Fig. 1) and imaged with a microscope objeclive (magnification 40 ) on a CCD camera. In order to prevent the camera to be damaged by the intense laser light, the transmitted and scattered laser light is blocked by a filter. The obrained data were recorded on tapes through a video systern which was connected to a computer for further analysis.

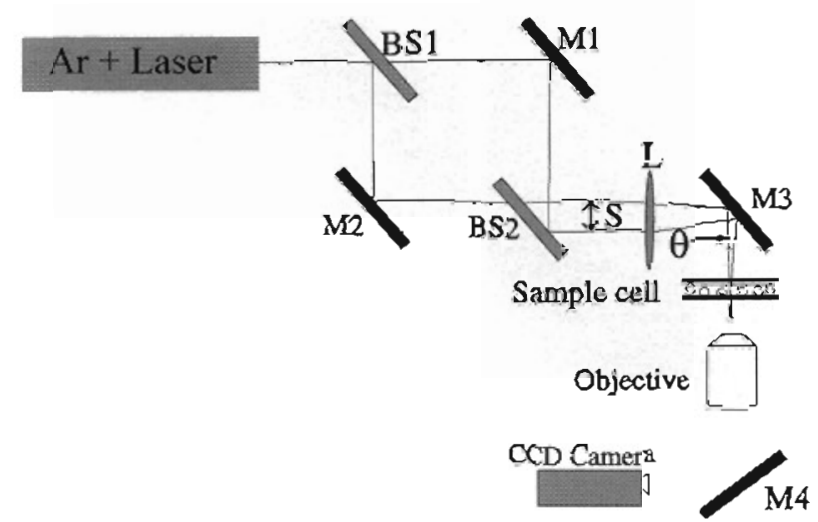

Fig. 1 The optical setup used during the experiments. M1, M2, M3, M4 are mirrors, BS1, BS2 beam splitters, $L$ is a lens. The position of mirror M2 which can be changed by a motor-controlled translation slage determines the fringe spacing $d$

\section{Results}

When the laser is switched on and the colloidal suspension is subjected to an intererence pattern, the radiation pressure causes the particles to be pushed lowards the bottom glass plate and a two-dimensional system is produced. Since glass surfaces are known to be negatively charged when immersed in water, the particles are prevented from sticking to the glass surface by electrostatic repulsion [15]. Due to the difference in the refraction indices of PS $\left(n_{\mathrm{p}}=1.59\right)$ and water $\left(n_{\mathrm{w}}=1.33\right)$ the particles are drawn into the intensity maximum of the interference grid which can be considered as an external periodic polential. The form of this potential $V(x)$ can be written as [11]

$V(x)=V_{0} \cos (2 \pi x / d)$,

where $\quad V_{0}=\left[3 n_{\mathrm{w}} \mathrm{Pr}^{3}\left(n^{2}-1\right) / c \sigma_{0}^{2}\left(n^{2}+2\right)\right]\left[j_{1}(\pi r / d) / 2 \pi r\right]$, with $P$ being the laser power, $c$ the light velocity in vacuum, $n=n_{\mathrm{p}} / n_{\mathrm{w}_{\mathrm{x}}} j_{1}$ the first-order spherical Bessel function, $r$ the particle diameter, and $\sigma_{0}$ the waist radius of the laser beam in the sample. Due to the Gaussian shape of the interfering laser beams, $V_{0}$ has also an Gaussian envelope. To minimize this effect [9] which would complicate the analysis we expanded the interference region to an area of about $300 \mu \mathrm{m}$ in diameter.

Figure 2a shows a typical configuration of particles in the sampe cell when no interference pattern is present. The particles are arranged as expected for a colloidal liquid and interacr only via a screened Coulomb potential. The corresponding Fourier transformation which is plotted in Fig. $2 b$ consists of two ring-shaped areas, the larger one being due to an illustration artefact, whereas the smaller (and darker) one confirms - due 10 the absence on any 

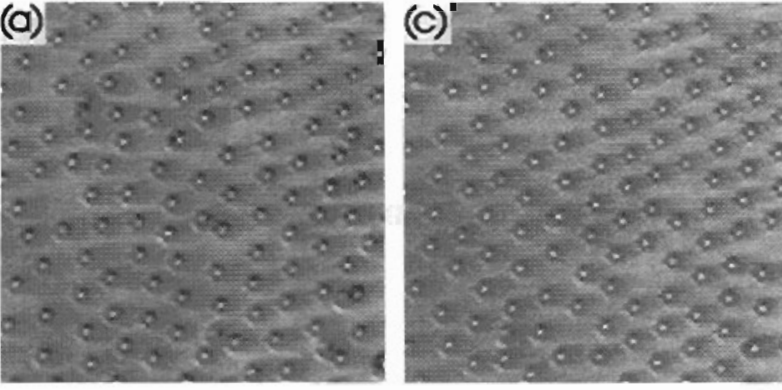

(b)

(d)
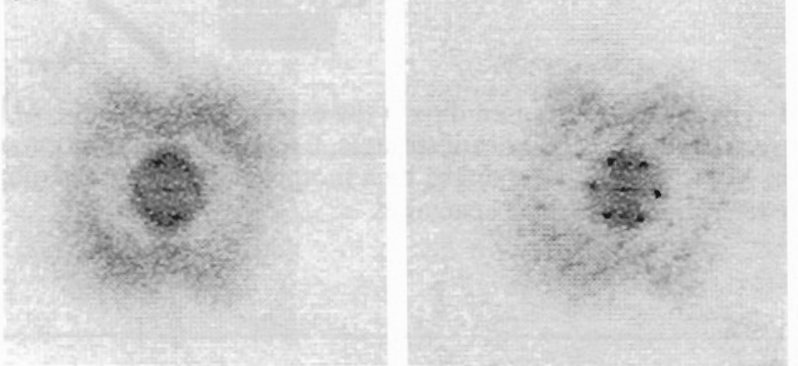

Fig. 2 Microscopic pictures and corresponding Fourier transiormation of colloidal structure (aj), (b) in absence of laser field, and (c), (d) when exposed to an interlerence pattern (laser intensity $200 \mathrm{~mW}$ ). The direction of the interfering fringes is vertical

distinct fealures - the liquid structure of Fig. 2a. When the laser is turned on and the interference pattern (the fringes are aligned vertically) interacts with the particles, the structure changes and starts to crystallize. This can be seen in Fig. 2c, where the laser light intensity is $200 \mathrm{~mW}$, corresponding to a potential depth of $1.9 \mathrm{k}_{\mathrm{B}} T$, and the fringe spacing $d=\sqrt{3 a} / 2$, with $a=10 \mu \mathrm{m}$ being the average particle distance determined from Fig. 2a. Under these conditions the interference fringes are commensurate with a hexagonal lattice which can be also seen in the Fourier iransformation in Fig. 2c.

However, when the modulation periodicity $d \neq$ $\sqrt{3 a} / 2$, deviation from a hexagonal symmetry are expected. Figure 3 shows several structures formed under different fringe spacing conditions, the laser light intensity was kept constant at a value of $200 \mathrm{~mW}$ as above. The ion concentration, i.e. the ionic conductivity during the experiments was kept contants at a value of $0.5 \mu \mathrm{S} / \mathrm{cm}$. When $d$ is increased (from the left to the right) the corresponding Fourier transformations clearly indicate that a change of the induced structure from a crystalline (Fig. $3 a$ and b) into a liquid-like structure (Fig. $3 \mathrm{~g}$ and h) occurs. With $a_{1}$ being the mean distance of particles along a row (parallel to the interference fringes) we can define the parameter $k=d / a_{1}$. As mentioned above, the close packed hexagonal lattice corresponds to $k=\sqrt{3} / 2=0.866$. When $k$ is smaller than that value, as being the case in Fig. $3 a$ and $b$, where $k$ was chosen to be 0.55 , the particles in adjacent rows are so close that a crystal with almost quadratic symmetry (which can be also considered as a hexagonal lattice distorted in the vertical direction) is observed. In fact, for $k=0.5$ we

Fig. 3 Microscopic picures and corresponding Fourier transformation for different fringe spacing. The ratio of the fringe spacing to the mean parcicle separation $a_{1}$ is $0.55 \mathrm{in} \mathrm{(a)} \mathrm{and} \mathrm{(b).} 0.91 \mathrm{in}(\mathrm{c})$ and (d), $1.0 \mathrm{in}(\mathrm{c})$ and (f), and $1.2 \mathrm{in}(\mathrm{g})$ and (h). The laser intensity is $200 \mathrm{mw}, t h e$ direcion of the fringes is vertical
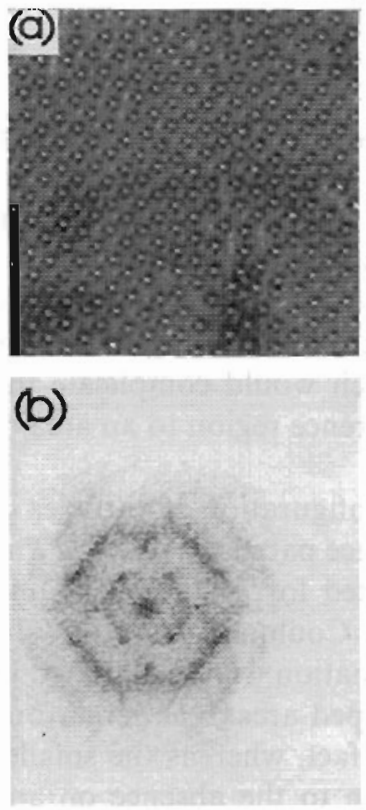

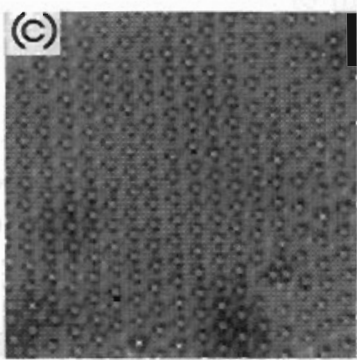

(d)

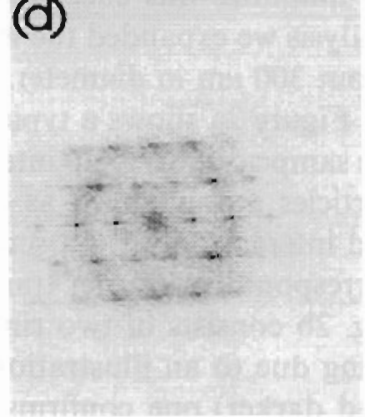

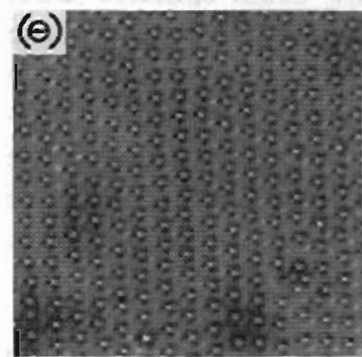

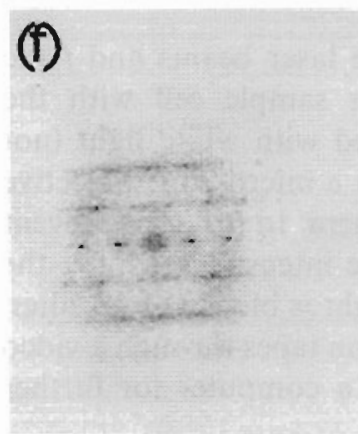

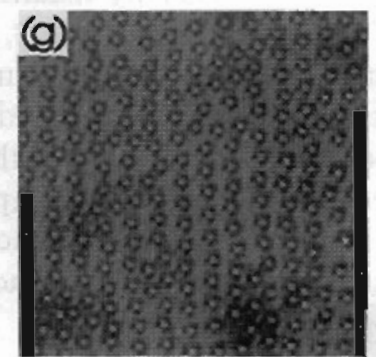

(h) 

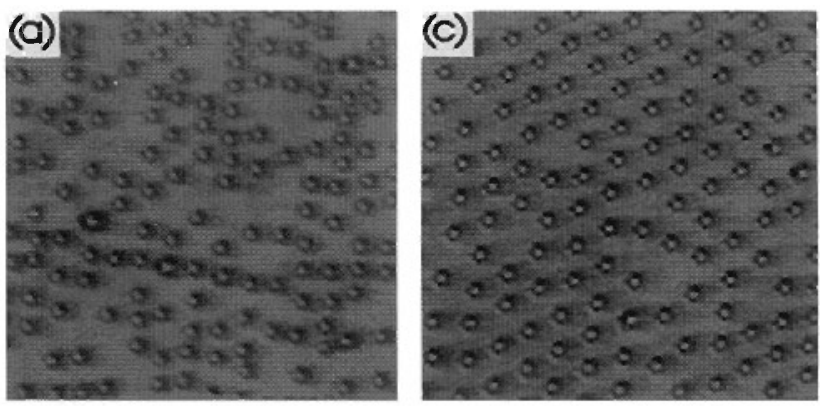

(b)

(d)

Fig. 4 Microscopic pictures and corresponding Fourier 1ransformation of colloids for two different ion concentrations. The conductivity of the colloidal suspension is 2.6 and $0.5 \mu \mathrm{S} / \mathrm{cm}$ respoctively, in (a) and (b) and (c) and (d). The laser intensity is $200 \mathrm{~mW}$ in both cases

observed a structure with exact quadratic synmetry. The structure in Fig. $3 c$ and $f$ with $k=0.91$, being very close to 0.866 , is nearly a hexagonal lattice. Wilh $k$ increased further 10 1.0, we oblain the siruclure shown in Fig. 3e and $\mathrm{g}$ which is still a hexagonal lattice but now distorted in the horizontal direction. However, as can be seen from the spots in the corresponding Fourier Lransformation (Fig. 3f), registration between neighboring rows (i.e. repulsive inferaction between particles of adjacent rows) still occurs. Figure $3 \mathrm{~g}$ and h finally, show the structure for $k=1.2$. The spots in Fig. 3h only correspond to a particle density modulation along vertical direction, but the registration between neighboring rows is lost because their interaction is smaller than the thermal energy. The obtained structure is a modulated liquid.

Finally, we want to demonstrate the effect on the light-induced structures when changing the ionic concenIration in the system. We found that for low particle density or high ion concentration where the system is far from the freezing condition, the colloidal liquids never freeze to a crystalline phase, even at very high light intensities. This can be seen from Fig. 4, where the particle concentrations are nearly the same (about $9 \%$ higher in Fig. 4a), but the ion concentration is changed. The measured conduclivity is 2.6 and $0.5 \mu \mathrm{S} / \mathrm{cm}$, respectively, for Fig. 4a and c. In Fig. 4a we only observe the alignment of particles along the interference fringes, however, no order within rows and no registration between them are found. This can be also seen in the corresponding Fourier transformation in Fig. 4b, which is characteristic of a modulated liquid. In contrast to this, after the ionic concentration was decreased, the colloidal suspension is crystallized under the influence of the same periodic light potential (Fig. $4 \mathrm{c}$ and d). This is in agreement to theoretical calculations [13]

In summary, we have studied the phase transitions of colloids under the influence of a periodic light field. We observed the light-induced freezing transition of the system when the fringe spacing and the particle concentration is chosen properly. Additionally, we also observed strong deviations of the induced crystal structure from a perfect hexagonal symmetry when the fringe spacing is veried. Finally, we demonstrated the infiuence of the salt concentration on LIF.

Acknowledgments Financial support by the Deutsche Forschungsgemeischafi. Sonderforschunggsbereich 513 is gratefully acknowledged. One of the authors (Q.H.W.) would like to acknowledge the research fellowship support from the Alexander von Humboldt Foundation.

\section{References}

1. Ashkin A (1970) Phy's Rev Lell 24:156; Aslzkin A, Dziedzic JM( (1975) Science 187:1073

2. Kepler GM, Fraden S (1994) Phys Rev Lett 73:356

3. Crocker JC, Grier DG (1996) Phys Rev Letl 73:352

4. Ashkin A, Schüize K, Dricdric JM, Eu. teneuer U, Schliwa M (1990) Nature $348: 346$

5. Block SM, Goldstein LSB, Schnapp BJ (1990) Nature 348:348
6. Austin RH. Brody JP, Cox EC, Duke T, Volkinuih W (1997) Phys Today 33 and reference therein

7. Bariv R, Moses E (1994) Phys Rev Lelt 73:1392

8. For a review, see Annu Rev Biophys Biomol Strucl (1994) 23:247

9. Chowdhury A Ackerson BJ. Clark NA (1985) Phys Rev Lell 60:833; Ackerson BJ, Chowdhury AH (1987) Faraday Discuss Chem Soc 83:309

10. Burns MM, Fournier J-M, Golovchenko JA (1990) Science 249:749
11. Loudiyi K, Ackerson BJ (1992) Physica 184A:I: 26

12. Chakraba1i J, Krishmamurliy HR, Sood AK (1994) Phys Rev Lett 73:2923

13. Chakrabati J, Krishnamurihy HR, Sood AK, Sengupta S (1995) Phys Rev Leit 75:2233

14. Pálberg $T$, Härıl W, Wittig U, Versmold H, Würth M (1992) J Phys Chem 96:8081

15. Prieve DC, Luo F, Lanni F (1987) Faraday Discus Chern Soc 83:297 